\section{Les besoins}

d'apprentissage

de la personne

atteinte de cancer:

une analyse
conceptuelle

Par Gemma Aucoin-Gallant

\section{Abrégé}

Cet article présente une analyse conceptuelle de l'expression besoin d'apprentissage d'une population atteinte de cancer. Cette analyse est effectuée à partir des étapes proposées par Wilson (I969). Afin de bien saisir les traits essentiels du concept besoin d'apprentissage, il est défini en précisant ses caractéristiques: les savoirs, les savoir-faire, les savoir-être et la notion d'apprendre. Par souci de clarification, l'expression besoin d'apprentissage a été analysée en examinant un groupe de concepts voisins: besoin d'information, désir d'apprendre et recherche d'information. Bien que souvent utilisée, la frontière existant entre ces concepts n'est pas clairement précisée. Il arrive que certains de ces concepts sont même confondus, L'article se termine en expliquant la nature des besoins d'apprentissage de la personne atteinte de cancer.

L'analyse conceptuelle est un exercice intellectuel qui cherche à découvrir la signification d'un concept en le distinguant des termes voisins et en précisant comment il est utilisé dans une situation particulière (Elias et Merriam, 1980). Ainsi, l'infirmière clarifie le concept étudié en se basant sur un processus de détermination, de ressemblance et de dissemblance (Boulanger et Goulet, 1994). Dans ses écrits, Wilson (1969) propose huit étapes relatives à l'analyse d'un concept. Selon lui, le concept est d'abord sélectionné et identifié. Puis, les caractéristiques sur lesquelles repose l'existence même du concept sont expliquées. La construction d'un cas modèle ct celle d'autres cas apportent une compréhension additionnelle au concept. Ensuite, les antécédents et les conséquents du concept sont présentés. Finalement, les référents empiriques permettent de rendre opérationnelles les caractéristiques du concept.

Le but de cet article est de préciser la nature et l'essence du concept besoin d'apprentissage concernant une population atteinte de cancer. $\mathrm{A}$ partir des étapes d'analyse proposées par Wilson, il est possible de clarifier la signification accordée à ce concept et d'offrir aux infirmières une manière d'évaluer objectivement les besoins d'apprentissage de la personne atteinte de cancer.

\section{Précision du concept besoin d'apprentissage Sélection du concept}

Dans la littérature en soins infirmiers, les termes besoin d'apprentissage, besoin d'information, désir d'apprendre et recherche d'information sont souvent utilisés et certains d'entre eux sont même confondus. La frontière existant entre ces concepts n'est pas clairement délimitée. Une consultation des banques de données informatisées en soins infirmiers et en éducation révèle qu'il n'existe aucune analyse conceptuelle de l'expression besoin d'apprentissage. Afin de remplir efficacement son rôle d'accompagnement lors d'une situation d'apprentissage, l'infirmière doit posséder une vision claire du concept besoin d'apprentissage pour mieux l'operationnaliser.

\section{Définition du concept}

Préoccupée par une vision holistique de la personne malade ou en santé, Henderson (1966) considère le besoin d'apprendre comme un besoin fondamental. En se basant sur le modèle conceptuel de Henderson en ce qui a trait aux soins infirmiers, Adam (1991) mentionne que le besoin d'apprendre commun à tous est celui "de découvrir, de satisfaire sa curiosité et d'ajouter à ses connaissances" (p.20). Adam (1991) précise également que chaque personne a un besoin d'apprendre qui lui est spécifique. Ainsi, les besoins d'apprendre peuvent varier d'un individu à un autre.

Selon son modèle d'adaptation, destiné aux soins infirmiers, Roy (1984) précise que l'être humain acquiert par l'apprentissage "des idées, des émotions et des croyances sur lui-même" (p.214). Dans une perspective de "caring", Watson (1985) mentionne que l'infirmière doit connaître la perception qu'a la personne de son besoin d'apprendre. Dans le domaine des soins infirmiers, l'accent est placé sur I'importance d'identifier le besoin d'apprentissage chez la personne et de le combler. En conséquence, le concept besoin d'apprentissage mérite d'être analysé de manière à le rendre mesurable.

De tous les théoriciens et penseurs qui se sont intéressés au phénomène de l'apprentissage, seul Knowles $(1980 ; 1990)$ a décrit l'apprentissage de l'adulte lié à des problèmes existentiels. Leader dans le domaine de l'andragogie, Knowles a différencié l'apprentissage à l'âge adulte de celui qui est propre aux jeunes apprenants. Selon lui, l'apprentissage de l'adulte est davantage orienté vers la résolution des problèmes de la vie quotidienne que sur un contenu à apprendre. Ainsi, l'adulte apprend afin de répondre à ses besoins actuels. Knowles $(1980 ; 1990)$ précise que le besoin d'apprentissage de l'adulte correspond à un écart à combler entre ce qu'il sait à un moment donné et ce qu'il souhaiterait apprendre afin d'améliorer son efficacité et sa qualité de vie. Cette définition précise d'abord les acquis de départ de l'apprenant: les savoirs, les savoir-faire et les savoir-être et elle implique aussi la notion

\section{ABSTRACT}

\section{THE LEARNING NEEDS OF THE CANCER PATIENT: A CONCEPTUAL ANALYSIS}

This paper offers a conceptual analysis of the term "learning need" of a cancer population. This analysis was made according to the steps recommended by Wilson (1969). In order to properly understand the essential features of the learning need concept, it is defined by focusing on its characteristics: cognitive knowledge, affective knowledge, psychomotor knowledge and the notion of learning. For the sake of clarity, the expression "learning need" was analyzed by examining a group of closely related concepts: information need, desire to learn and search for information. Although it is often used, the boundary between these concepts is not clearly identified. Some of these concepts may even be confused for each other. The paper concludes by explaining the nature of the cancer patient's learning needs. 
d'apprendre. Il importe maintenant de présenter ces caractéristiques sur lesquelles reposent l'existence même du concept besoin d'apprentissage.

\section{Détermination des caractéristiques}

Définir le concept besoin d'apprentissage suppose que l'on explique les termes savoir, savoir-faire et savoir-être ainsi que la notion d'apprendre. Selon Marchand (1985), l'apprentissage est un changement intentionnellement recherché par l'adulte qui se produit lors de l'acquisition de certains savoirs, savoir-faire et savoir-être. Ainsi, la personne intègre un certain nombre de savoirs, de savoir-faire et de savoir-être qui composent son savoir-devenir (De Ketele, Chastrette, Cross, Mettelin et Thomas, 1989).

Utilisé seul, le mot savoir signifie l'ensemble des connaissances acquises ou approfondies par une personne (De Ketele et al., 1989). Le savoir se rapporte à "l'aspect cognitif" et il représente "le côté théorique de l'apprentissage" (Marchand, 1985, p.112). Nullement opposé au savoir, le savoir-faire est "la capacité de la personne de structurer ses actions" (Reboul, 1980, p.68). Il implique alors "I'acquisition ou l'approfondissement d'opérations, de techniques et de moyens" (Marchand, 1985, p.112). Devant des habiletés à accomplir, le savoir-faire se démontre par l'exécution d'une tâche avec aisance, dextérité et précision (Reboul, 1980). Le savoir-faire éduque la personne tout entière et celle-ci apprend par le fait même à être. Le savoir-être implique alors des changements d'attitudes, de valeurs et une croissance personnelle qui s'oriente vers l'actualisation de soi (Marchand, 1985).

Après avoir expliqué clairement les termes savoir, savoir-faire et savoir-être, il importe de préciser la notion d'apprendre. Selon Doucet (1992), apprendre c'est découvrir, c'est acquérir de nouvelles connaissances et de nouveaux moyens, et être en mesure de les utiliser dans d'autres tâches. Dans le même ordre d'idées, Reboul (1980) souligne qu'apprendre "c'est d'abord déchiffrer et découvrir dans I'aspect des choses" (p.29); c'est aussi "la capacité de la personne de structurer ses actions... et de les transférer dans une situation donnée" (p.68). Reboul (1980) se base sur les constructions syntaxiques du verbe apprendre et il met en évidence les résultats de son analyse. Selon lui, "apprendre que" est "un acte d'information et son résultat est le renseignement" (p.9). Ainsi, la personne apprend qu'elle est atteinte de cancer. Reboul mentionne ensuite qu" "apprendre à" est "un acte d'apprentissage et son résultat est le savoir-faire" (p.10). Par exemple, la personne atteinte de cancer apprend à reconnaître les symptômes associés à la chimiothérapie. Les deux sens attribués par Reboul (1980) au terme apprendre nous amènent à préciser comment le concept besoin d'apprentissage peut être utilisé dans une situation concrète,

\section{Construction du cas modèle}

Walker et Avant (1995) mentionnent que le cas modèle représente un exemple typique du concept étudié. Ces deux auteurs précisent en effet, que "the model case should be a pure case of the concept" (p.42). Le récit ci-dessous d'une personne atteinte de cancer illustre l'utilisation du concept besoin d'apprentissage dans une situation concrète. Madame X mentionne:

\section{Je n'ai pas été informée des types de ressources disponibles dans mon milieu pour $m$ 'aider. J'aimerais bien les connâtre. Je ne sais pas comment expliquer à mes enfants ma maladie. Personne ne $m$ 'a rien dit. J'aimerais être informée à ce sujet. J'aimerais aussi apprendre à être plus positive devant ma situation de maladie.}

Les verbatimes présentés indiquent clairement que Madame $\mathrm{X}$ a des besoins d'apprentissage. Elle perçoit d'abord qu'elle n'a pas été informée de l'existence des ressources disponibles dans le milieu; de la manière d'expliquer à ses enfants sa maladie et de la façon d'être plus positive face au cancer. Madame X désire aussi recevoir de I'information à cet effet. Elle souhaite acquérir des savoir, des savoir-faire et des savoir-être. Dans le modèle présenté, il existe un écart entre la perception d'avoir été informé et celle de désirer de l'information. Il y a donc un besoin d'apprentissage. Même si l'information est au service de l'apprentissage (Reboul, 1988), il ne faut pas déduire qu'elle représente un besoin d'information pour la personne. De plus, si la personne atteinte de cancer mentionne qu'elle n'a pas été informée cela ne signifie pas que l'infirmière ne lui a pas dispensé de l'enseignement mais bien que la personne perçoit qu'il y a pas eu apprentissage.

\section{Construction d'autres cas}

La construction d'autres cas apporte une compréhension additionnelle au concept étudié et prévient toute ambiguinté quant à sa signification (Walker et Avant, 1995). Dans l'analyse du concept, le cas relié est celui qui "présente de nombreuses similitudes avec le concept étudié" (Boulanger et Goulet, 1994, p.47). À titre d'exemple, si Madame X souhaite connaître les dates prévues pour les traitements de radiothérapie prescrits. Il s'agit là d'un cas relié c'est-à-dire qu'il fait état d'un besoin d'information demandé par la personne atteinte de cancer. Comme les concepts besoin d'information et besoin d'apprentissage présentent des ressemblances importantes, il importe de les distinguer clairement.

Dans un contexte éducatif, le besoin d'apprentissage permet à la personne de préciser ce qu'elle veut acquérir au niveau des savoirs, des savoir-faire et des savoir-être. Le besoin d'apprentissage engage alors la personne tout entière. Le besoin d'information signifie une exigence chez l'individu d'obtenir "un élément de connaissances qu'il ne possédait pas et ne pouvait pas trouver en lui-même" (Reboul, 1980, p.12). Les besoins d'information relèvent alors du domaine du renseignement et indiquent bien les besoins d'"apprendre que" (Reboul, 1980). Ils se situent au plus bas niveau de l'acte d'apprendre. Dans la hiérarchie des comportements cognitifs, le besoin de connaître un nouveau fait relève de la connaissance. Celle-ci représente le comportement le plus simple (Bloom, Engelhart, Furst, Hill et Krathwohl, 1969). Ainsi, le besoin d'information fait abstraction des autres besoins de savoir de la personne. Il exclut aussi les besoins de la personne au niveau des savoir-faire et des savoir-être. II apparaît évident que les besoins d'apprentissage incluent les besoins d'information, mais l'inverse n'est pas vrai (Reboul, 1980).

En anglais, l'expression "learning needs" correspond à besoins d'apprentissage (Malcuit et Pomerleau, 1977; Levasseur-Ouimet et Tardif, 1983). Dans le domaine des soins infirmiers, les termes "learning needs" et "informational needs" sont souvent utilisés et même confondus. La frontière existant entre ces deux expressions n'est pas clairement précisée. Dans ses écrits, Derdiarian (1987; 1989) a privilégié le terme "informational needs" sans toutefois l'expliquer. S'agit-il des besoins se rapportant au savoir "knowing that" et/ou au savoir-faire "knowing how"? Derdiarian (1987) présente cependant la définition de "need for information". Selon cette auteure, "need for information" indique "le manque de connaissances, des connaissances imprécises ou incomplètes" (p. 157). Cette définition plutôt brève tend à se rapprocher de l'expression besoin d'information. Toutefois, Derdiarian (1987) précise que son instrument intitulé "Derdiarian Informational Needs Assessment (DINA)" permet au sujet d'indiquer l'information désirée. Est-ce que l'information désirée par les personnes atteintes de cancer se rapporte seulement à l'acquisition de connaissances ou vise-t-elle plutôt l'acquisition de certains savoirs, savoir-faire ou savoir-être?

Dans sa recherche effectuée auprès de 60 adultes atteints de cancer, Derdiarian (1987) a utilisé son échelle intitulée DINA. Les résultats de cette étude démontrent que $68 \%$ des sujets voulaient savoir comment faire face aux perturbations émotionnelles et $41 \%$ d'entre cux désiraient savoir comment composer avec le diagnostic de cancer ou cours de la communication entre les époux. Les deux exemples cités font ressortir que l'échelle DINA permet d'identifier et de décrire les besoins d'apprentissage. II faut toutefois se rappeler que les besoins d'information se limitent "à un élément de connaissances que l'individu ne possédait pas et ne pouvait pas trouver en lui-même" (Reboul, 1980, 
p.19). Or, vouloir savoir comment faire face aux perturbations émotionnelles ne soulève pas de la connaissance mais il s'agit bien pour I'individu d'acquérir des savoir-faire et des savoir-être. II apparaît évident que l'instrument DINA permet de décrire les besoins d'apprentissage d'une population atteinte de cancer. En s'appuyant principalement sur les écrits de Reboul (1980), il a été possible de distinguer clairement entre les besoins d'apprentissage et ceux d'information. Les antécédents et les conséquents du concept besoin d'apprentissage font partie de la prochaine étape de l'analyse conceptuelle.

\section{Identification des antécédents \\ et des conséquents}

Les antécédents d'un concept sont les éléments qui précèdent normalement l'existence du concept (Boulanger et Goulet, 1994). Le besoin d'apprentissage peut avoir comme préalables, la personne atteinte de cancer est capable d'indiquer à l'infirmière si elle a été informée et si elle désire de l'information en terme de savoir, savoir-faire et savoir-être. Les préalables au concept besoin d'apprentissage peuvent être ressortis afin de clarifier davantage la portée de ce concept.

Dans les écrits consultés, les expressions désir d'apprendre et recherche d'information sont souvent utilisés de manière équivalente au point de les présenter comme des synonymes. En effet, l'étude de Cassileth, Zupkis, Sutton-Smith et March (1980) représente un tel exemple. Ces auteurs se servent des expressions désirer apprendre et rechercher de l'information de façon indifférenciée. Il est donc important de clarifier ces deux termes. Dans un contexte éducatif, le désir d'apprendre est "une tendance devenue consciente" d'une nécessité à apprendre qui pousse la personne à agir. Le désir d'apprendre implique alors la prise de conscience par une personne de sa nécessité à apprendre, mais il n'inclut pas la mise en action (Sillamy, 1989). Ainsi la personne atteinte de cancer peut désirer apprendre mais elle n'entreprend aucune action.

La recherche d'information vient du désir d'apprendre. Elle est une démarche intentionnelle de la part de la personne exigeant de celle-ci un effort personnel et une action en vue de lui permettre d'acquérir certains savoirs, savoir-faire et savoir-être. Selon Bélanger (1992), la recherche d'information représente "une démarche à risque" pour la personne cancéreuse et elle se réalise dans un "contexte interactionnel et organisationnel" (p.53-54). Cette démarche à risque s'explique par le fait que la personne malade peut obtenir des informations susceptibles de lui rappeler la souffrance ou la mort. Toujours selon Bélanger (1992), la recherche d'information se caractérise par un processus de "sélection continuelle de l'information" afin de permettre à la personne cancéreuse de se "construire une réalité tournée vers la vie, de la sauvegarder et de l'optimaliser"

(p.52). La recherche d'information est alors l'actualisation du désir d'apprendre (Bélanger, 1992). Comme la recherche d'information prend habituellement naissance dans le cadre d'un contexte interactionnel, cela suppose que la personne atteinte de cancer et l'infirmière entrent en interaction à un moment donné. La personne atteinte de cancer peut alors exprimer ses besoins d'apprentissage.

Contrairement aux antécédents, les conséquents sont des éléments qui succèdent au concept étudié (Boulanger et Goulet, 1994). Ce sont des phénomènes qui naissent et qui peuvent être observés à la suite de l'expression d'un besoin d'apprentissage. D'abord, la personne atteinte de cancer perçoit inévitablement une insatisfaction concernant son besoin d'apprentissage. Cette insatisfaction fait appel à des réactions affectives tels le mécontentement et le déplaisir qui incitent la personne à mobiliser des énergies, de manière consciente ou non consciente en vue de combler le besoin (Sillamy, 1989). En présence d'un besoin d'apprentissage identifié, l'infirmière dispense de l'enseignement. De concert avec la personne soignée, l'infirmière organise alors des activités d'apprentissage en vue de favoriser l'acquisition de savoir, savoir-faire et savoir-être.

\section{Référents empiriques}

Les référents empiriques permettent d'opérationnaliser les caractéristiques d'un concept (Boulanger et Goulet, 1994). Tout en

Tableau 1: Catégories des besoins d'apprentissage proposées par Derdiarian (1987)

Catégories des besoins d'apprentissage

A. Première catégorie des besoins: le problème de santé

B. Deuxième catégorie des besoins: les préoccupations personnelles

C. Troisième catégorie des besoins: les préoccupations familiales

D. Quatrième catégorie des besoins: les préoccupations sociales
Liste des besoins d'apprentissage

1. Besoin d'apprentissage à propos du diagnostic médical

2. Besoin d'apprentissage à propos des examens cliniques

3. Besoin d'apprentissage à propos du traitement

4. Besoin d'apprentissage à propos du pronostic

5. Besoin d'apprentissage à propos de la carrière

6. Besoin d'apprentissage concernant la planification et les buts futurs

7. Besoin d'apprentissage concernant le bien-être physique

8. Besoin d'apprentissage concernant le bien-être psychologique

9. Besoin d'apprentissage concernant les rôles habituels

10. Besoin d'apprentissage des enfants concernant la maladie de leur père ou mère

11. Besoin d'apprentissage des parents concernant la maladie de leur fils ou fille

12. Besoin d'apprentissage des personnes significatives de la personne malade

13. Besoin d'apprentissage concernant les relations au travail

14. Besoin d'apprentissage à propos des relations avec ses amis

15. Besoin d'apprentissage à propos du besoin d'affiliation

16. Besoin d'apprentissage concernant les autres relations. 
conservant l'essence du concept étudié, il est possible de le rendre mesurable. Il est justifié de préciser que le besoin d'apprentissage correspond à un écart entre la perception d'avoir été informé et celle de désirer de l'information.

Seule Derdiarian $(1987,1989)$ a regroupé en catégories les besoins d'apprentissage de la personne atteinte de cancer. II s'agit des besoins d'apprentissage liés au problème de santé, aux préoccupations personnelles et aux préoccupations familiales ainsi que sociales. La première catégorie de besoins précise que la personne atteinte de cancer doit être informée au sujet de la nature de son problème de santé, des examens cliniques, du traitement et du pronostic. La deuxième catégoric concerne le besoin d'apprentissage ressenti par la personne malade au sujet de ses préoccupations face à son travail rémunéré et par rapport à sa vie personnelle. La troisième catégorie de besoins reliée à l'enseignement désiré par rapport aux préoccupations familiales vécues au sein de la famille. Finalement, la quatrième catégorie de besoins spécifie que la personne atteinte de cancer désire être informée au sujet des préoccupations vécues quant à sa vie sociale. Derdiarian $(1987 ; 1989)$ décrit avec précision les besoins d'apprentissage de la personne en se souciant du problème de santé vécu et de son retentissement sur les plans personnel, familial et social.

Le tableau 1 illustre les quatre catégories de besoins d'apprentissage de la personne atteinte de cancer décrits par Derdiarian (1987; 1989). Chaque catégorie englobe quatre besoins d'apprentissage distincts. Ce tableau précise, par exemple, les quatre différents besoins d'apprentissage de la personne atteinte de cancer concernant son problème de santé. II s'agit du besoin d'apprendre à propos: 1. du diagnostic médical; 2 . des examens cliniques; 3 . du traitement et 4 . du pronostic.

En se basant sur la définition opérationnelle du concept besoin d'apprentissage, deux questions sont suffisantes pour identifier le besoin actuel d'apprentissage de la personne atteinte de cancer. Ces deux questions sont les suivantes:

1. Avez-vous été informé au sujet de?

2. Quelle information aimeriez-vous recevoir à propos de?

\section{Références}

Adam, E. (1991). Être infirmière: un modèle conceptuel. Montréal: Études Vivantes.

Bélanger, S. (1992). Le processus de la recherche d'information de personnes atteintes de cancer. Mémoire de maîtrise inédit. Montréal: Université de Montréal.

Bloom, B.S., Engelhart, M.D., Furst, E.J., Hill, W.H. et Krathwohl, D.R. (1969). Taxonomie des objectifs pédagogiques, tome 1. Domaine cognitif. Montréal: Éducation Nouvelle.

Boulanger, J., et Goulet, C. (1994). Mieux faire connaissance dès la naissance. L'infirmière canadienne, 90 (4), 44-48.

Cassileth, B.R., Zupkis, R.V., Sutton-Smith, K., et March, V. (1980). Information and participation preferences among cancer patients. Annals of Internal Medicine, 92(6), 832-836.

De Ketele, J.M., Chastrette, M., Cross, P., Mettelin, P., et Thomas, J. (1989). Guide du Formateur. Bruxelles: De Boeck-Wesmael.

Derdiarian, A.K. (1987). Informational needs of recently diagnosed cancer patients: Part II. Method and description. Cancer Nursing, 10(3), 156-163.

Derdiarian, A.K. (1989). Effects of information on recently diagnosed cancer patients' and spouses' satisfaction with care. Cancer Nursing, 12(5), 285-292.

Doucet, J.J. (1992). Apprendre à apprendre. Caraquet: Franc-Jeu. Elias, J.L., et Merriam, S. (1980). Penser l'éducation des adultes. Montréal: Guérin.

Henderson, V. (1966). The nature of nursing. New York: Macmillan. Knowles, M.S. (1980). The modern practice of adult education: Andragogy versus pedagogy. New York: Association Press.
Ces deux questions peuvent être formulées pour chacun des besoins d'apprentissage identifiés par Derdiarian (Voir tableau 1). Elles déterminent de plus s'il existe un écart entre la perception d'avoir reçu de l'information et celle de désirer de l'information. Reboul (1980) précise que la personne qui n'a pas la compétence voulue pour recevoir l'information perçoit, à ce moment-là, qu'il n'a pas été informé. Par contre, l'adulte qui a su intégrer l'information reçue perçoit qu'il y a eu apprentissage. La deuxième question ci-haut mentionnée receuille les informations désirées par la personne atteinte de cancer et obtient les données préférentielles. Selon Nadeau (1988) cette procédure est celle qui est le plus fréquemment utilisée pour identifier les besoins d'apprentissage d'une population.

\section{Conclusion}

L'article avait pour but de présenter une analyse conceptuelle de l'expression besoin d'apprentissage concernant les personnes atteintes de cancer. Selon les théoriciens en soins infirmiers et en andragogie, les termes savoir, savoir-faire et savoir-être ainsi que la notion d'apprendre constituent principalement les caractéristiques du concept étudié. Par souci de clarification, l'expression besoin d'apprentissage a été analysée en examinant un groupe de concepts voisins. Ainsi, le désir d'apprendre et la recherche d'information sont des préalables au concept besoin d'apprentissage et ils représentent alors les antécédents du concept étudié. La notion d'insatisfaction et l'enseignement sont des phénomènes qui sont observés à la suite des besoins d'apprentissage et ils constituent les conséquents du concept.

Au terme de l'analyse conceptuelle, il se dégage que le besoin d'apprentissage correspond à un écart à combler entre la perception qu'a la personne d'avoir été informée et celle de désirer de l'information. Dans la pratique, les infirmières peuvent utiliser les deux questions qui découlent de la définition opérationnelle retenue dans l'article et cela, pour chacun des besoins d'apprentissage identifié par Derdiarian. Ainsi, les infirmières seront en mesure d'évaluer les besoins d'apprentissage de la personne atteinte de cancer.

Knowles, M.S. (1990), L'apprenant adulte: vers un nouvel art de la formation. Paris: Éditions d'organisation.

Levasseur-Ouimet, R, et Tardif, C. (1983). Lexique des termes techniques de l'éducation et de la pédagogie. Alberta: Université de l'Alberta.

Malcuit, G., et Pomerleau, A. (1977). Terminologie en conditionnement et apprentissage. Montréal: Presses de l'Université du Québec.

Marchand, L. (1985). Introduction à l'éducation des adultes. Boucherville: Gaëtan Morin.

Nadeau, M.A. (1988). L'évaluation de programme: théorie et pratique. Québec: Presses de l'Université Laval.

Orlando, I.J. (1961). The dynamic nurse-patient relationship. New York: Putnam's Sons.

Reboul, O, (1980). Qu'est-ce-qu'apprendre? Paris: Presses Universitaires de France.

Roy, C. (1984). Introduction aux soins infirmiers: un modèle de l'adaptation. Chicoutimi: Gaëtan Morin.

Sillamy, N. (1989). Dictionnaire de la psychologie. Paris: Larousse.

Walker, L.O., et Avant, K.C. (1995). Strategies for theory construction in nursing. Norwalk: Appleton \& Lange.

Watson, J. (1985). Nursing: Human sciences and human care, Norwalk: Appleton-Century-Crofts.

Wilson, J. (1969). Thinking with concepts. New York: Cambridge University Press. 